\title{
Transverse vibrations of double-tapered cantilever beams with end support and with end mass
}

\author{
H. H. Mabie \\ Virginia Polytechnic Institute and State University, Blacksburg, Virginia 24061
}

C. B. Rogers

Sandia Corporation, Albuquerque, New Mexico 87115

(Received 24 September 1973)

\begin{abstract}
The free vibrations of a double-tapered cantilever beam with (1) end support and (2) end mass have been investigated using the Bernoulli-Euler equation. The beam was tapered linearly in the horizontal and in the vertical planes simultaneously with the taper ratio in the horizontal plane equal to that in the vertical plane. A table is presented for the first case from which the fundamental frequency, second, third, fourth, and fifth harmonic can easily be obtained for various taper ratios. A chart, plotted from this table, shows the effect of taper ratio on the various harmonics. For the second case, a table and resulting charts show the effect of taper ratio and ratio of end mass to beam mass on the fundamental frequency and higher harmonics. Although previously presented, the case of the beam with free end is also included for purposes of comparison.
\end{abstract}

Subject Classification: $\mathbf{4 0 . 2 2 .}$

\section{INTRODUCTION}

This analysis is a continuation of the work ${ }^{1-3}$ started by the authors several years ago on the vibration of tapered cantilever beams. This type of beam tapered linearly in either the horizontal or the vertical plane is widely used for electrical contacts and for springs in electro-mechanical devices. Occasionally, however, the desired spring rate cannot be achieved by tapering the beam in only one plane so that it is necessary to resort to a taper in the horizontal and in the vertical plane simultaneously. Both the single-tapered and the doubletapered beams will require an end mass or an end support depending upon whether the beam is used for an electrical contact (normally open) or for a spring. Singletapered cantilever beams with end mass and with end support have been treated by the authors. ${ }^{1,2}$

This paper deals with the vibration of double-tapered cantilever beams with end support and with end mass for the case where the taper ratio in the horizontal plane equals that in the vertical plane. Tables have been developed from which the fundamental frequency, second, third, fourth, and fifth harmonic can be obtained for various taper ratios and ratios of end mass to beam mass. Although the case of the double-tapered cantilever beam with free end has been presented in Ref. 3, it is included in this work for comparative purposes.

\section{BEAM OF LINEARLY VARIABLE THICKNESS AND OF LINEARLY VARIABLE WIDTH}

In Ref. 3 the differential equation of motion for a vibrating beam tapered in two planes as shown in Fig. 1 was developed from the Bernoulli-Euler equation

$$
\frac{\partial^{2}}{\partial x^{2}}\left(\frac{E I \partial^{2} y}{\partial x^{2}}\right)=-\left(\frac{\rho A}{g}\right) \frac{\partial^{2} y}{\partial t^{2}},
$$

where $\rho A / g$ is the mass per unit length ( $\rho$ weight density, $A$ cross-sectional area, $g$ gravitational constant), $E$ the modulus of elasticity, and $I$ the moment of inertia. A sustained free vibration at a frequency $\omega$ of $y(x, t)$ $=z(x) \sin \omega t$ was assumed which gave the following:

$$
\begin{aligned}
\frac{d^{4} z}{d u^{4}} & +\frac{2 d^{3} z}{d u^{3}}\left[\frac{3(\alpha-1)}{1+(\alpha-1) u}+\frac{\beta-1}{1+(\beta-1) u}\right]+\frac{6 d^{2} z}{d u^{2}} \\
& \times\left\{\frac{(\beta-1)(\alpha-1)}{[1+(\beta-1) u][1+(\alpha-1) u]}+\frac{(\alpha-1)^{2}}{[1+(\alpha-1) u]^{2}}\right\} \\
= & \frac{(l k)^{4} z}{[1+(\alpha-1) u]^{2}},
\end{aligned}
$$

where

$$
\begin{aligned}
& u=x / l, \\
& \alpha=h_{0} / h_{1}, \\
& \beta=b_{0} / b_{1}, \\
& k^{4}=12 \rho \omega^{2} / E g h_{1}^{2} .
\end{aligned}
$$
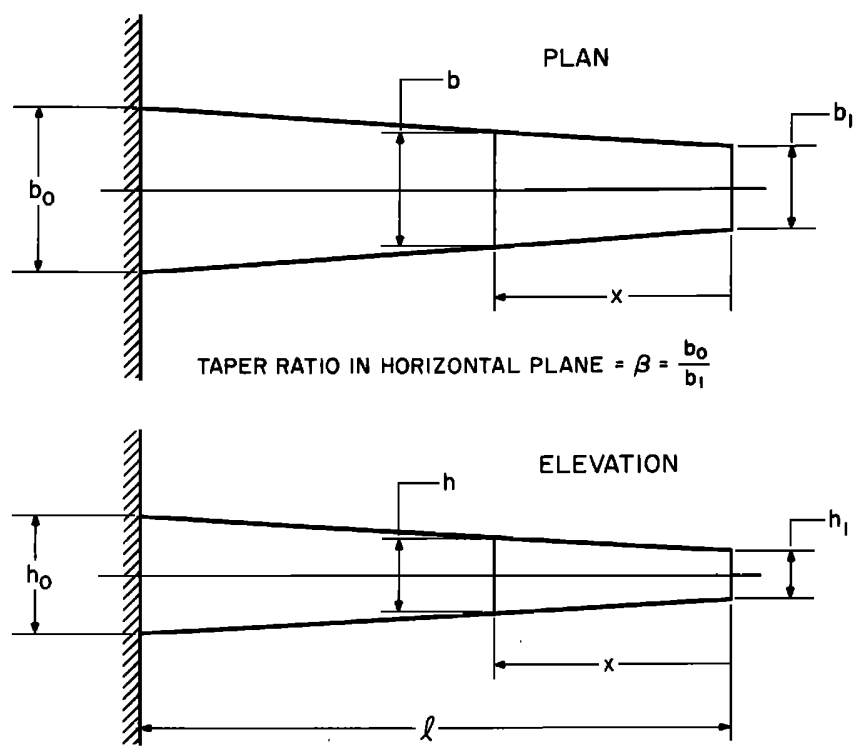

TAPER RATIO IN VERTICAL PLANE $=\alpha=\frac{h_{0}}{h_{1}}$

FIG. 1. Cantilever beam tapered linearly in horizontal and in vertical planes simultaneously. 


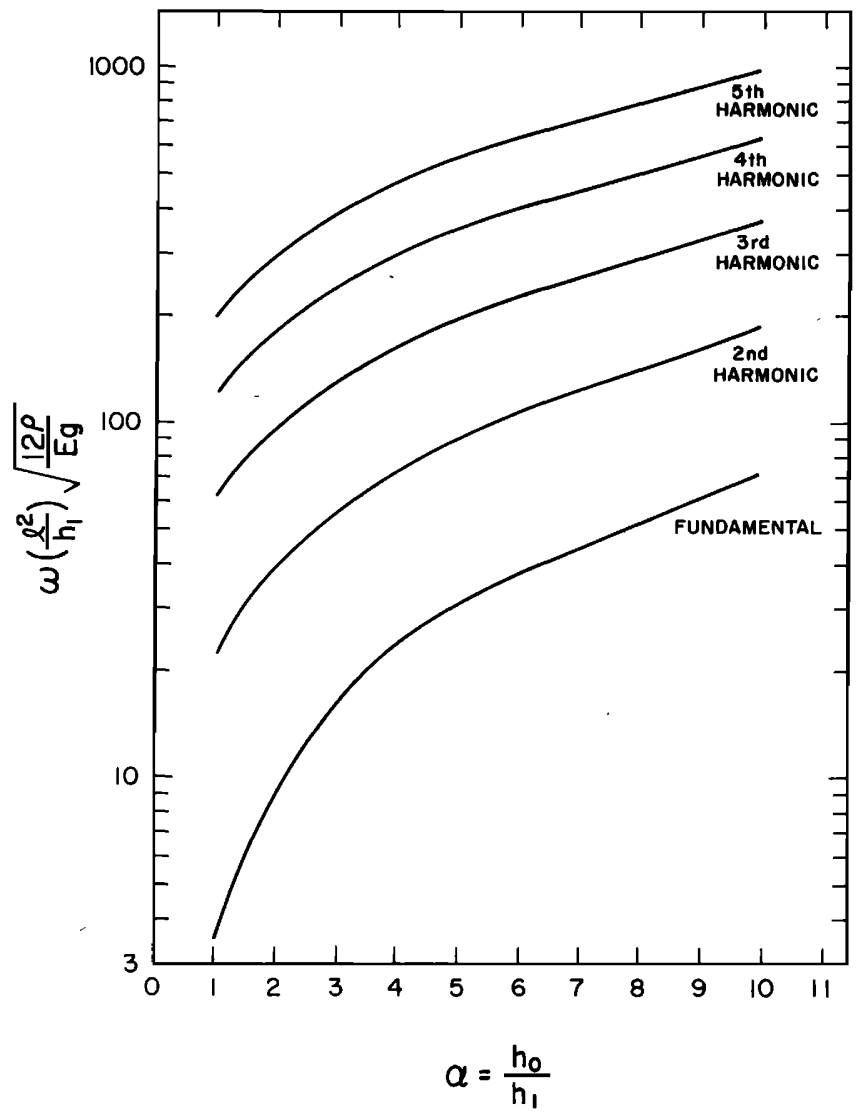

FIG. 2. Frequencies for double-tapered cantilever beam with free end with $\beta=\alpha$.

A formal solution for this equation could not be obtained, and it was solved by numerical integration to give values of $(l k)$ for various taper ratios of $\alpha$ and $\beta$.

A solution to Eq. 2 can be obtained by considering the special case where the taper ratios $\alpha$ and $\beta$ are equal.

The resulting differential equation can then be solved in terms of Bessel functions. If $\beta=\alpha$ is substituted in Eq. 2 , the following equation results:

$$
\begin{aligned}
\frac{d^{4} z}{d u^{4}} & +\frac{8 d^{3} z}{d u^{3}}\left[\frac{(\alpha-1)}{1+(\alpha-1) u}\right]+\frac{12 d^{2} z}{d u^{2}}\left[\frac{(\alpha-1)}{1+(\alpha-1) u}\right]^{2} \\
= & \frac{(l k)^{4} z}{[1+(\alpha-1) u]^{2}} .
\end{aligned}
$$

Equation 3 may be placed in a more recognizable form if $\phi=1+(\alpha-1) u$; this substitution yields

$$
\phi^{4} \frac{d^{4} z}{d \phi^{4}}+8 \phi^{3} \frac{d^{3} z}{d \phi^{3}}+12 \phi^{2} \frac{d^{2} z}{d \phi^{2}}=\left(\frac{l k}{\alpha-1}\right)^{4} \phi^{2} z .
$$

Siddall and Isackson ${ }^{4}$ list the steps to put Eq. 4 into operator notation for which Watson ${ }^{5}$ gives the solution as

$$
\begin{aligned}
z= & \frac{1}{\phi}\left[A J_{2}\left(\frac{2 l k}{\alpha-1} \sqrt{\phi}\right)+B Y_{2}\left(\frac{2 l k}{\alpha-1} \sqrt{\phi}\right)\right. \\
& \left.+C I_{2}\left(\frac{2 l k}{\alpha-1} \sqrt{ } \phi\right)+D K_{2}\left(\frac{2 l k}{\alpha-1} \sqrt{\phi}\right)\right],
\end{aligned}
$$

where $J_{2}$ and $Y_{2}$ are Bessel functions of the first and second kind and $I_{2}$ and $K_{2}$ are modified Bessel functions of the first and second kind.
TABLE I. Factor $(l k)^{2} \beta=\alpha$, free end.

\begin{tabular}{rccccc}
\hline \hline & $\begin{array}{l}\text { Funda- } \\
\text { mental } \\
\text { frequency }\end{array}$ & $\begin{array}{l}\text { Second } \\
\text { harmonic }\end{array}$ & $\begin{array}{l}\text { Third } \\
\text { harmonic }\end{array}$ & $\begin{array}{l}\text { Fourth } \\
\text { harmonic }\end{array}$ & $\begin{array}{l}\text { Fifth } \\
\text { harmonic }\end{array}$ \\
\hline & $\mathbf{3 . 5 1 6 0 2}$ & 22.03449 & $\mathbf{6 1 . 6 9 7 2 1}$ & 120.9019 & 199.8595 \\
1.0 & $\mathbf{4 . 5 4 9 9 7}$ & 25.46665 & $\mathbf{6 8 . 9 8 6 8 6}$ & 133.9884 & 220.6668 \\
1.4 & $\mathbf{5 . 6 4 8 2 8}$ & $\mathbf{2 8 . 8 7 8 4 8}$ & 76.14960 & 146.7374 & 240.8424 \\
1.6 & $\mathbf{6 . 8 0 2 2 1}$ & 32.28233 & 83.21828 & 159.2265 & 260.5263 \\
$\mathbf{1 . 8}$ & $\mathbf{8 . 0 0 4 7 8}$ & $\mathbf{3 5 . 6 8 6 1 5}$ & 90.21556 & 171.5096 & 279.8154 \\
2.0 & $\mathbf{9 . 2 5 0 3 0}$ & $\mathbf{3 9 . 0 9 5 2 3}$ & $\mathbf{9 7 . 1 5 7 8 0}$ & 183.6255 & 298.7798 \\
2.5 & $\mathbf{1 2 . 5 2 2 6}$ & $\mathbf{4 7 . 6 6 2 2}$ & 114.3459 & 213.3596 & 345.0885 \\
3.0 & $\mathbf{1 5 . 9 7 8 5}$ & 56.3146 & $\mathbf{1 3 1 . 3 8 6 2}$ & 242.5213 & 390.2255 \\
$\mathbf{3 . 5}$ & $\mathbf{1 9 . 5 7 8 1}$ & $\mathbf{6 5 . 0 6 5 5}$ & 148.3511 & 271.2901 & 434.5191 \\
$\mathbf{4 . 0}$ & $\mathbf{2 3 . 2 9 2 3}$ & $\mathbf{7 3 . 9 1 8 8}$ & 165.2854 & 299.7799 & 478.1801 \\
$\mathbf{5 . 0}$ & $\mathbf{3 0 . 9 8 2 0}$ & $\mathbf{9 1 . 9 2 7 3}$ & 199.1682 & 356.2088 & 564.1394 \\
$\mathbf{1 0 . 0}$ & $\mathbf{7 2 . 0 4 8 7}$ & $\mathbf{1 8 6 . 8 0 2}$ & 371.238 & 635.049 & 981.657 \\
\hline \hline
\end{tabular}

\section{A. Beam with free end}

For a beam with a free end at $x=0$, the boundary conditions are

$$
\begin{aligned}
& \text { at } x=0 \text { or } u=0, d^{2} z / d u^{2}=0 \text { and } d^{3} z / d u^{3}=0, \\
& \text { at } x=l \text { or } u=1, d z / d u=0 \text { and } z=0 .
\end{aligned}
$$

With these boundary conditions, the solution becomes that of a double-tapered cantilever beam which is truncated and tapers from the fixed end only. Imposing the above boundary conditions on the general solution in $\mathrm{Eq}$. 5 gives the following determinantal equation for obtaining the natural frequencies of the beam:

$$
\left|\begin{array}{llll}
J_{2}(\Theta \sqrt{\alpha}) & Y_{2}(\Theta \sqrt{\alpha}) & I_{2}(\Theta \sqrt{\alpha}) & K_{2}(\Theta \sqrt{\alpha}) \\
J_{3}(\Theta \sqrt{\alpha}) & Y_{3}(\Theta \sqrt{\alpha}) & -I_{3}(\Theta \sqrt{\alpha}) & K_{3}(\Theta \sqrt{\alpha}) \\
J_{4}(\Theta) & Y_{4}(\Theta) & I_{4}(\Theta) & K_{4}(\Theta) \\
J_{5}(\Theta) & Y_{5}(\Theta) & -I_{5}(\Theta) & K_{4}(\Theta)
\end{array}\right|=0
$$

The various values of $\Theta=[2 l k /(\alpha-1)]$ were found. To compare with values tabulated in Ref. 3, Table I was developed giving values of $(l k)^{2}$ corresponding to funda-

\begin{tabular}{|c|c|c|c|c|c|}
\hline$\alpha$ & $\begin{array}{l}\text { Funda- } \\
\text { mental } \\
\text { frequency }\end{array}$ & $\begin{array}{l}\text { Second } \\
\text { harmonic }\end{array}$ & $\begin{array}{l}\text { Third } \\
\text { harmonic }\end{array}$ & $\begin{array}{l}\text { Fourth } \\
\text { harmonic }\end{array}$ & $\begin{array}{l}\text { Fifth } \\
\text { harmonic }\end{array}$ \\
\hline 1.0 & 15.4182 & 49.9649 & 104.248 & 178.270 & 272.031 \\
\hline 1.2 & 17. 5615 & 55.4779 & 115.077 & 196. 337 & 299.264 \\
\hline 1.4 & 19.6505 & 60.8528 & 125.577 & 213.801 & 325.540 \\
\hline 1.6 & 21.6980 & 66.1184 & 135.817 & 230.790 & 351.061 \\
\hline 1. 8 & 23.7128 & 71.2951 & 145.847 & 247. 391 & 375.967 \\
\hline 2.0 & 25.7010 & 76.3976 & 155.702 & 263.671 & 400.361 \\
\hline 2.5 & 30.5821 & 88.8974 & 179.735 & 303.257 & 459.572 \\
\hline 3.0 & 35. 3696 & 101.117 & 203.108 & 341.627 & 516.841 \\
\hline 3.5 & 40.0904 & 113.129 & 225.989 & 379.089 & 572.656 \\
\hline 4.0 & 44.7611 & 124.980 & 248.488 & 415.841 & 627.331 \\
\hline 5.0 & 53.9936 & 148.315 & 292.615 & 487.727 & 734.077 \\
\hline 6.0 & 63.1233 & 171.289 & 335.881 & 558.001 & 838.218 \\
\hline 10.0 & 99.0859 & 261.086 & 503.841 & 829.474 & 1239.11 \\
\hline
\end{tabular}
mental, second harmonic, third harmonic, fourth harmonic, and fifth harmonic frequencies for the free-end case with $\beta=\alpha$. Rearranging the equation $k^{4}=12 \rho \omega^{2} /$ $E g h_{1}^{2}$ to obtain the term $(l k)^{2}$, it follows that

$$
\omega\left(l^{2} / h_{1}\right)(12 \rho / E g)^{1 / 2}=(l k)^{2} .
$$

TABLE II. Factor $(l k)^{2} \beta=\alpha$, end support. 


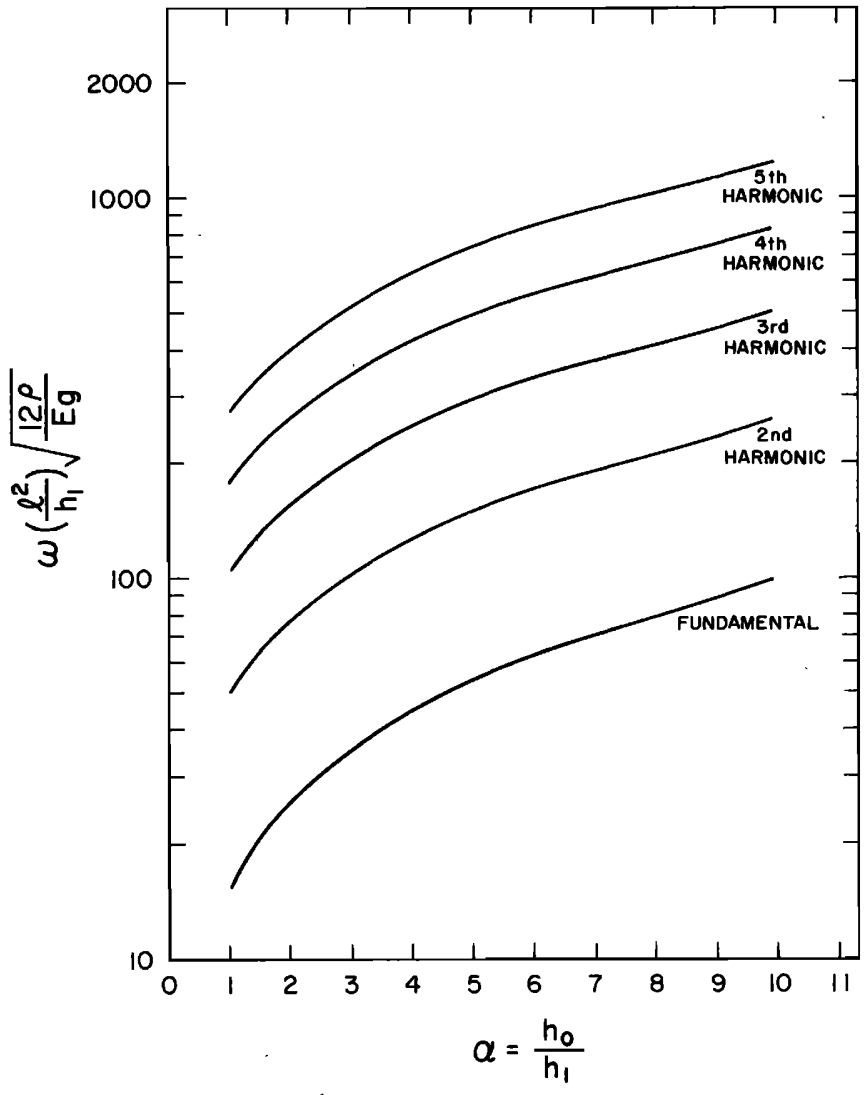

FIG. 3. Frequencies for double-tapered cantilever beam with end support with $\beta=\alpha$.

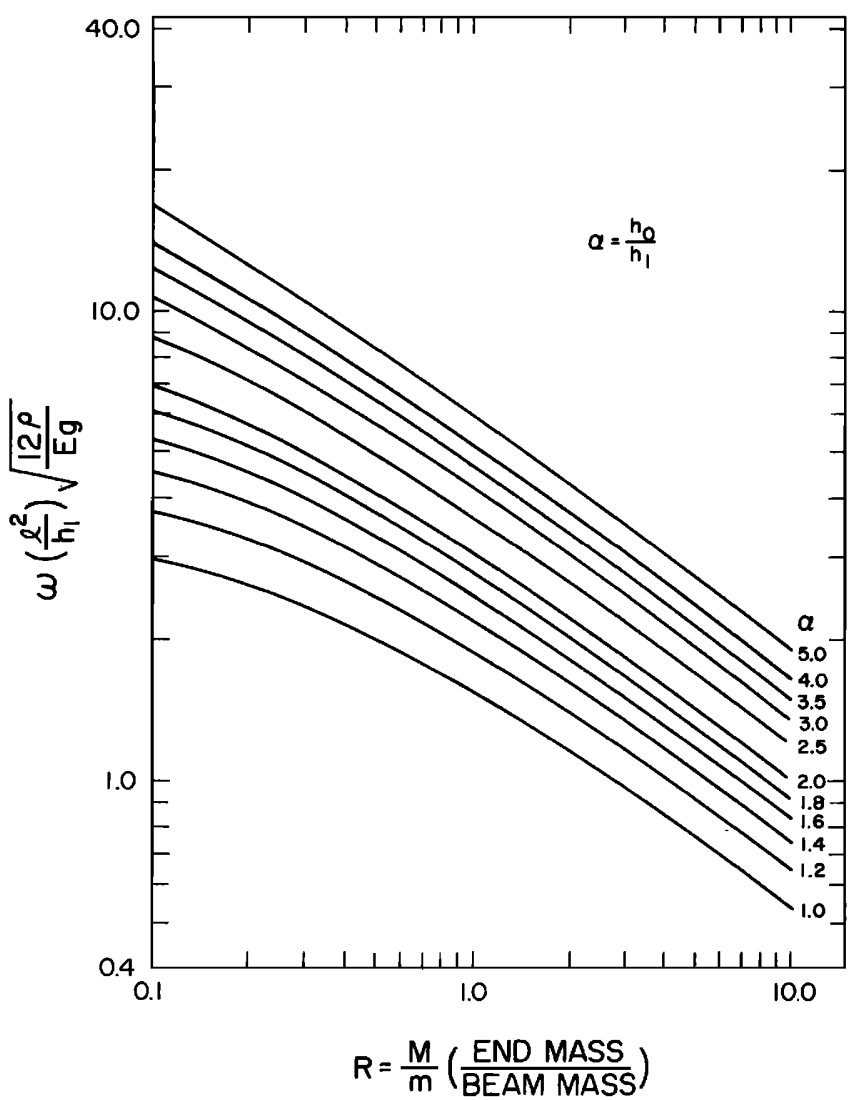

FIG. 4. Fundamental frequency for double-tapered cantilever beam with end mass with $\beta=\alpha$.

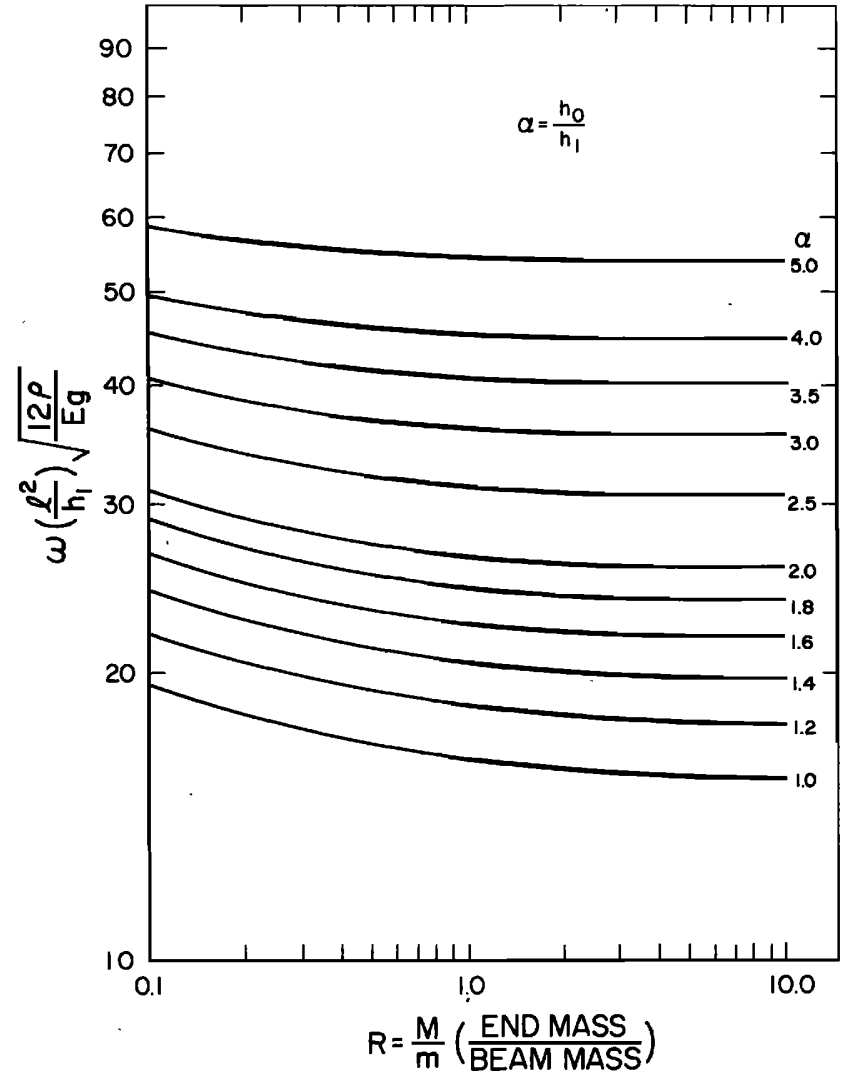

FIG. 5. Second-harmonic frequency for double-tapered cantilever beam with end mass with $\beta=\alpha$.

Using this equation and the values of $(l k)^{2}$ from Table I, curves were plotted of $\omega\left(l^{2} / h_{1}\right)(12 \rho / E g)^{1 / 2}$ vs $\alpha$ for the five harmonics as shown in Fig. 2.

\section{B. Beam with end support}

For a beam with end support at $x=0$, the boundary conditions are:

$$
\begin{aligned}
& \text { at } x=0 \text { or } u=0, d^{2} z / d u^{2}=0 \text { and } z=0 ; \\
& \text { at } x=l \text { or } u=1, d z / d u=0 \text { and } z=0 .
\end{aligned}
$$

Imposing these boundary conditions gives the following determinantal equation for obtaining the natural frequencies of the beam with end support:

$\left|\begin{array}{llcl}J_{2}(\Theta \sqrt{\alpha}) & Y_{2}(\Theta \sqrt{\alpha}) & I_{2}(\Theta \sqrt{\alpha}) & K_{2}(\Theta \sqrt{\alpha}) \\ J_{3}(\Theta \sqrt{\alpha}) & Y_{3}(\Theta \sqrt{\alpha}) & -I_{3}(\Theta \sqrt{\alpha}) & K_{3}(\Theta \sqrt{\alpha}) \\ J_{4}(\Theta) & Y_{4}(\Theta) & I_{4}(\Theta) & K_{4}(\Theta) \\ J_{2}(\Theta) & Y_{2}(\Theta) & I_{2}(\Theta) & K_{2}(\Theta)\end{array}\right|=0$.

Table II was developed to give values of $(l k)^{2}$ corresponding to the fundamental, second, third, fourth, and fifth harmonic frequencies for the end support case with $\beta=\alpha$. From Table II, curves were plotted of $\omega\left(l^{2} / h_{1}\right) \cdot$ $(12 \rho / E g)^{1 / 2}$ vs $\alpha$ for the five harmonics as shown in Fig. 3 .

\section{Beam with end mass}

For the case of a concentrated mass $M$ located at $x=0$, the boundary conditions are: 
TABLE III. Factor $(l k)^{2} \beta=\alpha$, end mass.

\begin{tabular}{|c|c|c|c|c|c|c|}
\hline$\alpha$ & $R$ & $\begin{array}{l}\text { Funda- } \\
\text { mental } \\
\text { frequency }\end{array}$ & $\begin{array}{l}\text { Second } \\
\text { harmonic }\end{array}$ & $\begin{array}{l}\text { Third } \\
\text { harmonic }\end{array}$ & $\begin{array}{l}\text { Fourth } \\
\text { harmonic }\end{array}$ & $\begin{array}{l}\text { Fifth } \\
\text { harmonic }\end{array}$ \\
\hline \multirow[t]{10}{*}{1.0} & .0 & 3. 51602 & 22.0345 & 61.6972 & 120.902 & 199.860 \\
\hline & .1 & 2.96784 & 19.3558 & 55.5182 & 110.708 & 185. 346 \\
\hline & .2 & 2.61275 & 18.2078 & 53.5586 & 108.192 & 182.431 \\
\hline & .4 & 2.16799 & 17.1763 & 52.0632 & 106.457 & 180.544 \\
\hline & .6 & 1. 89246 & 16.7007 & 51.4451 & 105.781 & 179.834 \\
\hline & .8 & 1. 70064 & 16.4274 & 51.1080 & 105.421 & 179.461 \\
\hline & 1.0 & 1. 55730 & 16.2501 & 50.8958 & 105.198 & 179.232 \\
\hline & 2.0 & 1.15820 & 15.8609 & 50.4476 & 104.735 & 178.760 \\
\hline & 5.0 & .756937 & 15.6024 & 50.1623 & 104.446 & 178.468 \\
\hline & 10.0 & .541375 & 15.5115 & 50.0644 & 104.347 & 178.369 \\
\hline \multirow[t]{10}{*}{1.2} & .0 & 4. 54997 & 25.4666 & 68.9869 & 133.988 & 220.667 \\
\hline & .1 & 3.75054 & 21.9023 & 61.1597 & 121.434 & 203.130 \\
\hline & .2 & 3. 25843 & 20.5336 & 59.0259 & 118.835 & 200.220 \\
\hline & .4 & 2. 66608 & 19.378́9 & 57.4964 & 117.138 & 198.423 \\
\hline & .6 & 2. 31036 & 18.8692 & 56.8871 & 116.496 & 197.763 \\
\hline & .8 & 2. 06697 & 18.5825 & 56.5601 & 116.159 & 197.420 \\
\hline & 1.0 & 1. 88708 & 18.3988 & 56.3562 & 115.951 & 197.210 \\
\hline & 2.0 & 1.39383 & 18.0022 & 55.9301 & 115.523 & 196.780 \\
\hline & 5.0 & .90655 & 17.7434 & 55.6620 & 115.257 & 196.516 \\
\hline & 10.0 & .64725 & 17.6534 & 55.5705 & 115.167 & 196.427 \\
\hline \multirow[t]{10}{*}{1.4} & .0 & 5. 64828 & 28.8785 & 76.1496 & 146.737 & 240.842 \\
\hline & .1 & 4. 54374 & 24.3118 & 66.5588 & 131.742 & 220.249 \\
\hline & .2 & 3.89751 & 22.7418 & 64.3072 & 129.126 & 217.404 \\
\hline & .4 & 3.14824 & 21.4913 & 62.7768 & 127.492 & 215.711 \\
\hline & .6 & 2.71077 & 20.9598 & 62.1850 & 126.887 & 215.100 \\
\hline & .8 & 2.41594 & 20.6662 & 61.8715 & 126.573 & 214.785 \\
\hline & 1.0 & 2.20010 & 20.4800 & 61.6773 & 126.380 & 214.593 \\
\hline & 2.0 & 1.61576 & 20.0832 & 61.2750 & 125.985 & 214.202 \\
\hline & 5.0 & 1. 04678 & 19.8282 & 61.0241 & 125.741 & 213.963 \\
\hline & 10.0 & .74633 & 19.7401 & 60.9389 & 125.659 & 213.882 \\
\hline \multirow[t]{10}{*}{1.6} & .0 & 6.80221 & 32.2823 & 83.2183 & 159.227 & 260.526 \\
\hline & .1 & 5. 33828 & 26.6067 & 71.7742 & 141.743 & 236.870 \\
\hline & .2 & 4. 52367 & 24.8590 & 69.4525 & 139.154 & 234.125 \\
\hline & .4 & 3.61163 & 23.5373 & 67.9436 & 137.595 & 232.540 \\
\hline & .6 & 3. 09243 & 22.9936 & 67.3741 & 137.028 & 231.976 \\
\hline & .8 & 2. 74709 & 22.6977 & 67.0753 & 136.735 & 231.687 \\
\hline & 1.0 & 2.49632 & 22.5117 & 66.8913 & 136.556 & 231.511 \\
\hline & 2.0 & 1. 82461 & 22.1195 & 66.5125 & 136.192 & 231.154 \\
\hline & 5.0 & 1.17831 & 21.8703 & 66.2779 & 135.968 & 230.936 \\
\hline & 10.0 & .83915 & 21.7848 & 66.1985 & 135.893 & 230.863 \\
\hline \multirow[t]{10}{*}{1.8} & .0 & 8.00478 & 35.6862 & 90.2156 & 171.510 & 279.815 \\
\hline & .1 & 6.12761 & 28.8055 & 76.8494 & 151.509 & 253.106 \\
\hline & .2 & 5. 13319 & 26.9054 & 74.4959 & 148.979 & 250.481 \\
\hline & .4 & 4.05537 & 25.5335 & 73.0228 & 147.498 & 249.000 \\
\hline & .6 & 3.45551 & 24.9849 & 72.4776 & 146.968 & 248.478 \\
\hline & .8 & 3. 06105 & 24.6901 & 72.1938 & 146.695 & 248.212 \\
\hline & 1.0 & 2.77658 & 24.5061 & 72.0199 & 146.529 & 248.051 \\
\hline & 2.0 & 2.02142 & 24.1213 & 71.6634 & 146.192 & 247.724 \\
\hline & 5.0 & 1.30198 & 23.8792 & 71.4439 & 145.986 & 247.525 \\
\hline & 10.0 & .92637 & 23.7965 & 71.3697 & 145.917 & 247.458 \\
\hline \multirow[t]{10}{*}{2.0} & .0 & 9.25030 & 39.0952 & 97.1578 & 183.626 & 298.780 \\
\hline & .1 & 6.90715 & 30.9239 & 81.8171 & 161.094 & 269.036 \\
\hline & .2 & 5. 72408 & 28.8964 & 79.4612 & 158.641 & 266.537 \\
\hline & .4 & 4. 47965 & 27.4920 & 78.0323 & 157.239 & 265.154 \\
\hline & .6 & 3.80088 & 26.9438 & 77.5118 & 156.742 & 264.671 \\
\hline & .8 & 3. 35894 & 26.6522 & 77.2427 & 156.488 & 264.425 \\
\hline & 1.0 & 3.04210 & 26.4714 & 77.0782 & 156.334 & 264.277 \\
\hline & 2.0 & 2.20735 & 26.0959 & 76.7426 & 156.021 & 263.976 \\
\hline & 5.0 & 1.41865 & 25.8613 & 76.5367 & 155.831 & 263.794 \\
\hline & 10.0 & 1.00862 & 25.7815 & 76.4674 & 155.767 & 263.733 \\
\hline \multirow[t]{10}{*}{2.5} & .0 & 12.52258 & 47.6622 & 114.3459 & 213.360 & 345.088 \\
\hline & .1 & 8.79472 & 35.9508 & 93.9102 & 184.480 & 307.844 \\
\hline & .2 & 7.11589 & 33.7041 & 91.6299 & 182.253 & 305.657 \\
\hline & .4 & 5.46099 & 32.2738 & 90.3256 & 181.032 & 304.485 \\
\hline & .6 & 4. 59487 & 31.7411 & 89.8638 & 180.609 & 304.082 \\
\hline & .8 & 4. 04183 & 31.4633 & 89.6277 & 180.394 & 303.878 \\
\hline & 1.0 & 3. 64982 & 31.2928 & 89.4842 & 180.263 & 303.755 \\
\hline & 2.0 & 2. 63178 & 30.9433 & 89.1934 & 180.001 & 303.507 \\
\hline & 5.0 & 1. 68463 & 30.7280 & 89.0164 & 179.842 & 303.358 \\
\hline & 10.0 & 1. 19606 & 30.6553 & 88.9570 & 179.788 & 303.308 \\
\hline 3.0 & .0 & 15.9785 & 56.3146 & 131. 386 & 242.521 & 390.225 \\
\hline & .1 & 10.5776 & 40.7106 & 105.707 & 207.291 & 345,583 \\
\hline & .2 & 8.38981 & 38.3575 & 103.559 & 205.290 & 343.670 \\
\hline & .4 & 6.34346 & 36.9508 & 102.377 & 204.222 & 342.665 \\
\hline & .6 & 5.30525 & 36.4437 & 101.966 & 203.856 & 342.323 \\
\hline & .8 & 4. 65153 & 36.1827 & 101.757 & 203.671 & 342.151 \\
\hline & 1.0 & 4.19184 & 36.0237 & 101.631 & 203.559 & 342.047 \\
\hline
\end{tabular}

TABLE III. (continued)

\begin{tabular}{|c|c|c|c|c|c|c|}
\hline$\alpha$ & $R$ & $\begin{array}{l}\text { Funda- } \\
\text { mental } \\
\text { frequency }\end{array}$ & $\begin{array}{l}\text { Second } \\
\text { harmonic }\end{array}$ & $\begin{array}{l}\text { Third } \\
\text { harmonic }\end{array}$ & $\begin{array}{l}\text { Fourth } \\
\text { harmonic }\end{array}$ & $\begin{array}{l}\text { Fifth } \\
\text { harmonic }\end{array}$ \\
\hline & 2.0 & 3. 00974 & 35.7003 & 101.376 & 203. 334 & 341.838 \\
\hline & 5.0 & 1. 92138 & 35.5028 & 101.221 & 203.199 & 341.712 \\
\hline & 10.0 & 1.36289 & 35.4363 & 101.169 & 203.153 & 341.670 \\
\hline \multirow[t]{10}{*}{3.5} & .0 & 19.5781 & 65.0655 & 148.351 & 271.290 & 434.519 \\
\hline & .1 & 12.2504 & 45.3044 & 117.324 & 229.703 & 382.541 \\
\hline & .2 & 9.55820 & 42.9227 & 115.324 & 227.905 & 380.856 \\
\hline & .4 & 7.14473 & 41.5643 & 114.252 & 226.962 & 379.983 \\
\hline & .6 & 5.94889 & 41.0857 & 113.883 & 226.641 & 379.687 \\
\hline & .8 & 5.20359 & 40.8416 & 113.697 & 226.479 & 379.539 \\
\hline & 1.0 & 4. 68250 & 40.6936 & 113.585 & 226.382 & 379.449 \\
\hline & 2.0 & 3. 35188 & 40.3942 & 113.358 & 226.186 & 379.269 \\
\hline & 5.0 & 2.13578 & 40.2124 & 113.221 & 226.068 & 379.161 \\
\hline & 10.0 & 1. 51400 & 40.1515 & 113.175 & 226.028 & 379.125 \\
\hline \multirow[t]{10}{*}{4.0} & .0 & 23.2923 & 73.9188 & 165.285 & 299.780 & 478.180 \\
\hline & .1 & 13.8168 & 49.7958 & 128.823 & 251.811 & 418.887 \\
\hline & .2 & 10.6353 & 47.4353 & 126.967 & 250.189 & 417.392 \\
\hline & .4 & 7. 87962 & 46.1360 & 125.990 & 249.348 & 416.623 \\
\hline & .6 & 6. 53887 & 45.6857 & 125.657 & 249.064 & 416.364 \\
\hline & .8 & 5.70965 & 45.4575 & 125.489 & 248.921 & 416.234 \\
\hline & 1.0 & 5.13237 & 45.3196 & 125.388 & 248.834 & 416.155 \\
\hline & 2.0 & 3.66580 & 45.0417 & 125.185 & 248.661 & 415.998 \\
\hline & 5.0 & 2. 33262 & 44.8736 & 125.062 & 248.557 & 415.904 \\
\hline & 10.0 & 1. 65278 & 44.8174 & 125.021 & 248.522 & 415.872 \\
\hline \multirow[t]{10}{*}{5.0} & .0 & 30.9820 & 91.9273 & 199.168 & 356.209 & 564.139 \\
\hline & .1 & 16.6644 & 58.6146 & 151. 581 & 295.344 & 490.174 \\
\hline & .2 & 12.5670 & 56.3745 & 149.980 & 293.999 & 488.964 \\
\hline & .4 & 9. 19450 & 55.1988 & 149.155 & 293.312 & 488.349 \\
\hline & .6 & 7. 59518 & 54.8000 & 148.877 & 293.081 & 488.142 \\
\hline & .8 & 6.61634 & 54.5994 & 148.737 & 292.965 & 488.038 \\
\hline & 1.0 & 5.93881 & 54.4788 & 148.653 & 292.895 & 487.976 \\
\hline & 2.0 & 4. 22934 & 54.2366 & 148.484 & 292.755 & 487.852 \\
\hline & 5.0 & 2.68636 & 54. 0909 & 148.382 & 292.671 & 487.777 \\
\hline & 10.0 & 1.90227 & 54.0423 & 148.348 & 292.643 & 487.752 \\
\hline
\end{tabular}

$$
\begin{aligned}
& \text { at } x=0, \partial^{2} y / \partial x^{2}=0 \text { and } \frac{\partial}{\partial x}\left(E I \frac{\partial^{2} y}{\partial x^{2}}\right)=-V=\frac{-M \partial^{2} y}{\partial t^{2}} ; \\
& \text { at } x=l, \partial y / \partial x=0 \text { and } y=0 .
\end{aligned}
$$

For the assumed free vibration $y(x, t)=z(x) \sin \omega t$, the boundary conditions at $x=0$ transform into

$$
d^{2} z / d x^{2}=0
$$

and

$$
d^{3} z / d x^{3}=M \omega^{2} z_{0} / E I_{0},
$$

where

$$
I_{0}=\frac{1}{12} b_{1} h_{1}^{3} .
$$

\section{Therefore,}

$$
\begin{aligned}
d^{3} z / d x^{3} & =12 M \omega^{2} z_{0} / E b_{1} h_{1}^{3} \\
& =\left(\frac{12 \rho \omega^{2}}{E g h_{1}^{2}}\right)\left(\frac{M z_{0} g}{\rho b_{1} h_{1}}\right) .
\end{aligned}
$$

The volume of the beam is $(l / 3) b_{1} h_{1}\left(\alpha^{2}+\alpha+1\right)$ so that the mass of the beam $m$ can be expressed as

$$
m=\left(\frac{\rho}{g}\right)\left[\left(\frac{l}{3}\right)\left(b_{1} h_{1}\right)\left(\alpha^{2}+\alpha+1\right)\right] .
$$


Therefore,

$$
\begin{aligned}
\frac{d^{3} z}{d x^{3}} & =\left(\frac{12 \rho \omega^{2}}{E g h_{1}^{2}}\right) \frac{M\left[\frac{1}{3} l\left(\alpha^{2}+\alpha+1\right)\right]}{(\rho / g) b_{1} h_{1}\left[\frac{1}{3} l\left(\alpha^{2}+\alpha+1\right)\right]} z_{0} \\
& =k^{4}(M / m)(l / 3)\left(\alpha^{2}+\alpha+1\right) z_{0} .
\end{aligned}
$$

Substituting $\phi=1+[(\alpha-1) / l] x$,

$$
\left(\frac{d^{3} z}{d \phi^{3}}\right)_{\phi=1}=k^{4}\left(\frac{M}{m}\right)\left(\frac{l^{4}}{3}\right)\left[\frac{\alpha^{2}+\alpha+1}{(\alpha-1)^{3}}\right](z)_{\phi=1} .
$$

The boundary conditions at $x=0$ or $\phi=1$ are therefore

$$
\frac{d^{2} z}{d \phi^{2}}=0
$$

and

$$
\frac{d^{3} z}{d \phi^{3}}=\left(\frac{M}{m}\right)\left[\frac{(l k)^{4}}{3}\right]\left[\frac{\alpha^{2}+\alpha+1}{(\alpha-1)^{3}}\right] z
$$

The boundary conditions at $x=l$ or $\phi=\alpha$ become

$$
z=0
$$

and

$$
\frac{d z}{d \phi}=0
$$

Imposing the boundary conditions given by Eqs. 9 on Eq. 5 gives the following determinantal equation for obtaining the natural frequencies of the beam with an end mass $M$ :

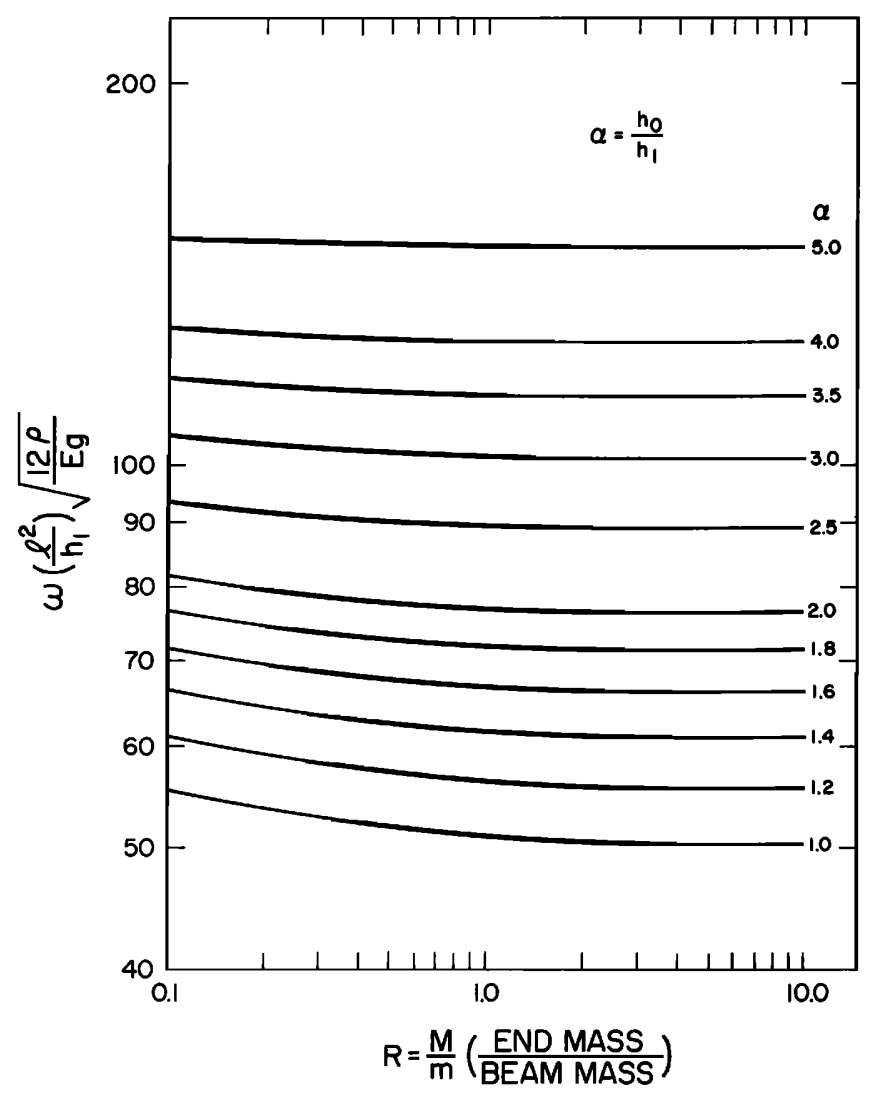

FIG. 6. Third-harmonic frequency for double-tapered cantilever beam with end mass with $\beta=\alpha$.

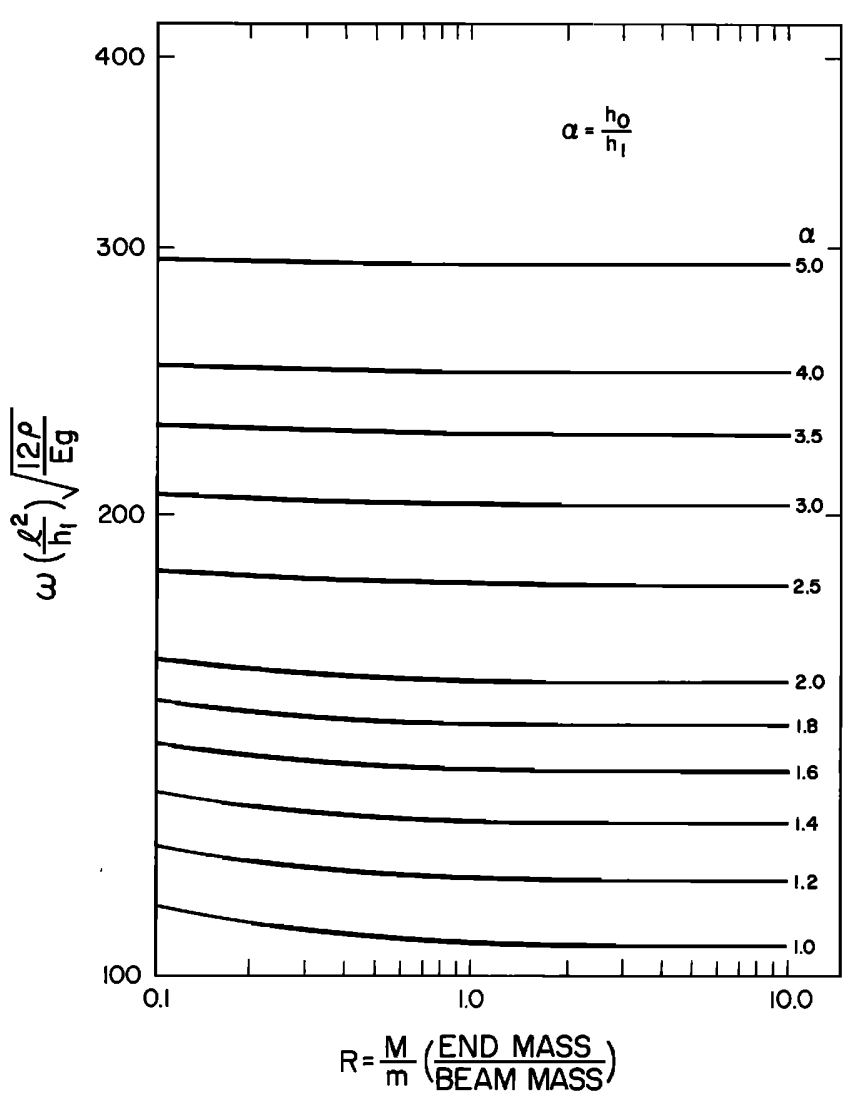

FIG. 7. Fourth-harmonic frequency for double-tapered cantilever beam with end mass with $\beta=\alpha$.

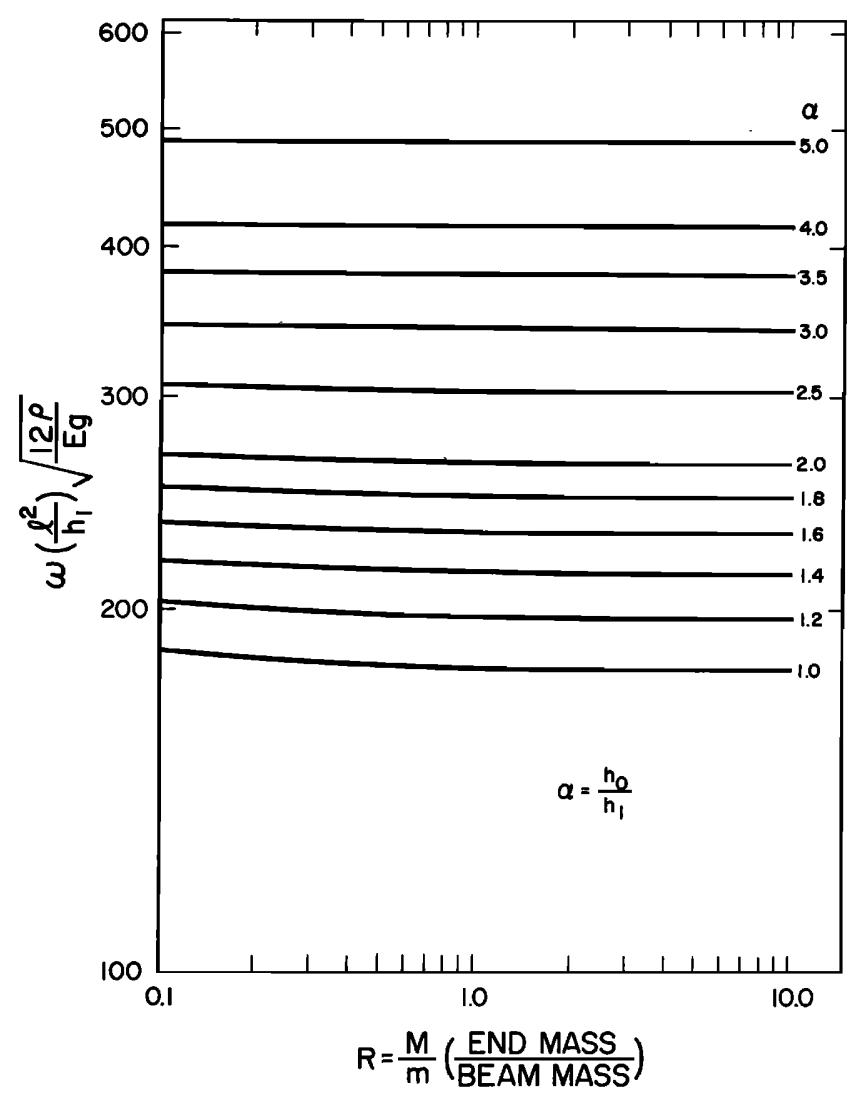

FIG. 8. Fifth-harmonic frequency for double-tapered cantilever beam with end mass with $\beta=\alpha$. 


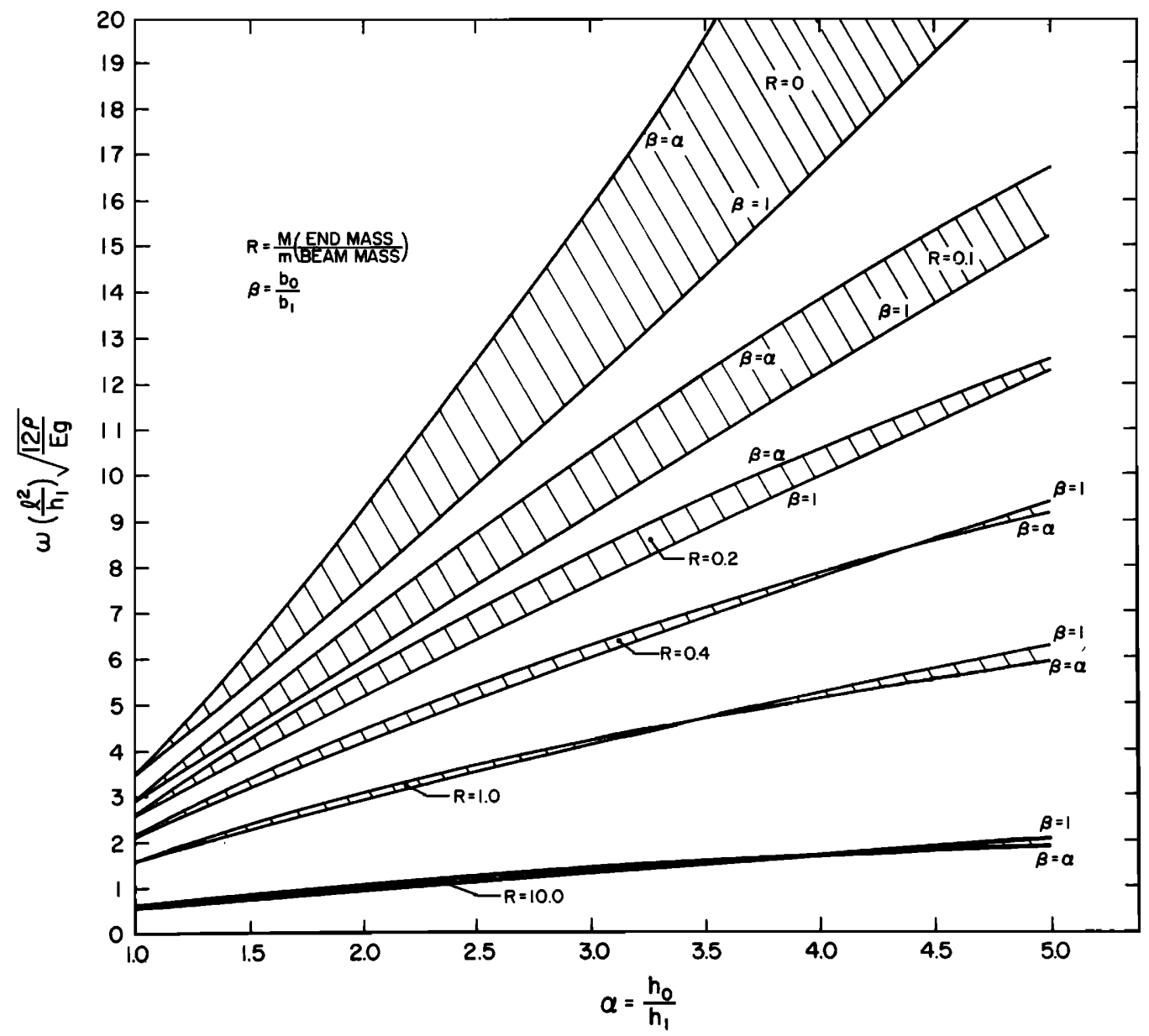

FIG. 9. Fundamental frequency for double-tapered cantilever beam with end mass with $\beta=\alpha$ and $\beta=1$ for several values of $R$.

$$
\left|\begin{array}{cccc}
J_{2}(\Theta \sqrt{\alpha}) & Y_{2}(\Theta \sqrt{\alpha}) & I_{2}(\Theta \sqrt{\alpha}) & K_{2}(\Theta \sqrt{\alpha}) \\
J_{3}(\Theta \sqrt{\alpha}) & Y_{3}(\Theta \sqrt{\alpha}) & -I_{3}(\Theta \sqrt{\alpha}) & K_{3}(\Theta \sqrt{\alpha}) \\
J_{4}(\Theta) & Y_{4}(\Theta) & I_{4}(\Theta) & K_{4}(\Theta) \\
A & B & C & D
\end{array}\right|=0 \text {, }
$$

where

$$
\begin{aligned}
& A:\left[\frac{M}{m}\right]\left[\frac{l k}{3}\right]\left(\alpha^{2}+\alpha+1\right) J_{2}(\Theta)+J_{5}(\Theta), \\
& B=\left[\frac{M}{m}\right]\left[\frac{l k}{3}\right]\left(\alpha^{2}+\alpha+1\right) Y_{2}(\Theta)+Y_{5}(\Theta), \\
& \left.\left.C=\left[\frac{M}{m}\right]\right] \frac{l k}{3}\right]\left(\alpha^{2}+\alpha+1\right) I_{2}(\Theta)-I_{5}(\Theta), \\
& D=\left[\frac{M}{m}\right]\left[\frac{l k}{3}\right]\left(\alpha^{2}+\alpha+1\right) K_{2}(\Theta)+K_{5}(\Theta) .
\end{aligned}
$$

Table III was developed to give values of $(l k)^{2}$ corresponding to the fundamental, second, third, 'fourth, and fifth harmonic frequencies for a beam with end mass with $\beta=\alpha$. In this table $R$ is the ratio $M / m$ of the mass of the concentrated load to that of the beam.

Figures 4-8 show curves of $\omega\left(l^{2} / h_{1}\right)(12 \rho / E g)^{1 / 2}$ vs $R$ for the five harmonics plotted from the data in Table III. It is interesting to note that the curves of $\omega\left(l^{2} / h_{1}\right)(12 \rho /$ $E g)^{1 / 2}$ vs $R$, after the fundamental frequency (Fig. 4 ), are almost independent of $R$. This is especially true for the higher harmonics and higher values of $\alpha$.

Figure 9 shows a plot of $\omega\left(l^{2} / h_{1}\right)(12 \rho / E g)^{1 / 2}$ vs $\alpha$ for the fundamental frequency for $\beta=\alpha$ and $\beta=1$ for several values of $R$. The values for $\beta=1$ were taken from the previously solved case given in Ref. 1 . It can be seen that as the value of $R$ increases, the spread of the two curves decreases for a particular $R$. This is particularly significant for the values of $R>0.4$. In these cases the value of the fundamental frequency is approximately the same whether the beam has a double taper or whether it tapers only in the vertical plane $(\beta=1)$.

${ }^{1}$ H. H. Mabie and C. B. Rogers, "Transverse Vibrations of Tapered Cantilever Beams With End Loads," J. Acoust. Soc. Am. 36, 463-469 (1964).

${ }^{2}$ H. H. Mabie and C. B. Rogers, "Transverse Vibrations of Tapered Cantilever Beams With End Support," J. Acoust. Soc. Am. 44, 1739-1741 (1968).

${ }^{3}$ H. H. Mabie and C. B. Rogers, "Transverse Vibrations of Double-Tapered Cantilever Beams," J. Acoust. Soc. Am. 51, 1771-1774 (1972).

${ }^{4} \mathrm{~J}$. N. Siddall and G. Isackson, "Approximate Analytical Methods for Determining Natural Modes and Frequencies of Vibration," MIT Rep. ONR Proj. NR-035-259, pp. 141-146 (Jan. 1951).

${ }^{5} \mathrm{G}$. N. Watson, A Treatise on the Theory of Bessel Functions (Cambridge U. P., Cambridge, England, 1952). 